\title{
EDUCAÇÃO ALIMENTAR NA ESCOLA: PARA ALÉM DA ABORDAGEM BIOLÓGICA
}

\author{
EDUCACIÓN ALIMENTARIA EN LA ESCUELA: ADEMÁS DEL \\ ENFOQUE BIOLÓGICO
}

\section{FOOD EDUCATION AT SCHOOL: BEYOND THE BIOLOGICAL APPROACH}

\author{
Mariana de Senzi ZANCUL ${ }^{1}$
}

RESUMO: O comportamento alimentar do ser humano não se resume à ingestão de alimentos, ou seja, comemos não apenas para manter as atividades vitais, mas recorremos às refeições como forma de obter prazer, propiciar a convivência e conter determinadas ansiedades, por exemplo. Portanto, a alimentação é um processo complexo, que ultrapassa a necessidade biológica e recebe influência de vários fatores sociais, culturais, geográficos, religiosos ou afetivos. No entanto, no que diz respeito ao espaço escolar, a Educação Alimentar, no Brasil, ainda é predominantemente estudada e praticada adotando-se a perspectiva da saúde e da doença. Aqui se pretendeu questionar essa situação e propor uma reflexão a respeito da maneira como a Educação Alimentar escolar vem sendo tratada, ressaltando a importância de atuações e práticas que extrapolem a abordagem exclusivamente biológica.

PALAVRAS-CHAVE: Educação e saúde. Educação alimentar. Alimentação.

RESUMEN: El comportamiento alimentario del ser humano no se reduce a la ingesta de alimentos, es decir, comemos no sólo para mantener las actividades vitales, pero recurrimos a las comidas como forma de obtener placer, propiciar la convivencia y contener ciertas ansiedades, por ejemplo. Por lo tanto, la alimentación es un proceso complejo, que sobrepasa la necesidad biológica y recibe influencia de varios factores sociales, culturales, geográficos, religiosos o afectivos. Sin embargo, en lo que se refiere al espacio escolar, la Educación Alimentaria, en Brasil, todavía es predominantemente estudiada y practicada adoptando la perspectiva de la salud y de la enfermedad. Aquí se pretendió cuestionar esa situación y proponer una reflexión acerca de la manera como la Educación Alimentaria escolar viene siendo tratada, resaltando la importancia de actuaciones y prácticas que extrapolen el enfoque exclusivamente biológico.

PALABRAS-CLAVE: Educación y salud. Educación alimentaria. Alimentación.

ABSTRACT: The feeding behavior of the human being is not limited to food intake, i.e., eat not only to maintain the vital activities, but we used to eat as a way to get pleasure, provide coexistence and contain certain anxieties. So the food is a complex process that

${ }^{1}$ Universidade de Brasília (UnB), Brasília - Brasil. Núcleo de Educação Científica, Instituto de Ciências Biológicas. E-mail: marianaib@unb.br. 
goes beyond the biological need and is influenced by various social, cultural, geographic, religious or emotional. However, with regard to the school environment, nutrition education, Brazil, is still predominantly studied and practiced from the standpoint of health and disease. This paper aims to question and propose a reflection on the way the Food Education school has been treated at school, stressing the importance of actions and practices that go beyond the purely biological approach.

KEYWORDS: Education and health. Food education. Nutrition.

\section{Introdução}

A alimentação é um dos fatores determinantes da saúde e qualidade de vida dos indivíduos. O tema alimentação é amplo e abordado por distintos profissionais, principalmente, em relação à promoção de hábitos alimentares saudáveis.

$\mathrm{O}$ ato de se alimentar é a primeira necessidade do ser humano sendo também considerado um prazer, indicando assim, sua dupla natureza. Como a respiração e o sono, o alimento faz parte dos imperativos humanos fundamentais e é também a expressão dos nossos desejos. Além de ser uma necessidade biológica, a alimentação inclui um complexo sistema simbólico de significados, como por exemplo; sociais, políticos, religiosos e éticos (CARNEIRO, 2003).

Segundo Castell (2004), o comportamento alimentar do ser humano não se resume à ingestão de alimentos, ou seja, comemos não apenas para manter as atividades vitais, mas é por meio das refeições que também obtemos prazer, convivemos em sociedade e praticamos as relações humanas. Por isso, a alimentação é um processo complexo, que ultrapassa a necessidade biológica e recebe influência de vários fatores emocionais, sociais, culturais, geográficos, religiosos ou afetivos.

A importância de uma boa alimentação tem sido bastante discutida em diversas esferas, destacando-se o espaço escolar. Os conteúdos relacionados à formação de hábitos em saúde fazem parte dos currículos escolares, tanto no Brasil como em outros países, e entre os objetivos da Educação Alimentar está o de preparar os indivíduos para escolhas alimentares saudáveis e de forma consciente (COELHO et al, 2008; LYNG, et. al, 2013; GREENWOOD e FONSECA, 2016).

Embora educar para a saúde seja responsabilidade de diferentes segmentos, a escola também é um ambiente fundamental de promoção da saúde (BRASIL, 1998). Soma-se a isso o fato de a escola ser um local em que crianças e adolescentes fazem refeições diariamente, realizando escolhas que revelam suas preferências e hábitos. 
No entanto, no que diz respeito ao espaço escolar, a Educação Alimentar, no Brasil, ainda é predominantemente estudada e praticada do ponto de vista da saúde e da doença, sob a perspectiva principalmente de nutricionistas, de médicos e de enfermeiros. Em estudos já publicados, os aspectos abordados referem-se, principalmente, a atuações pautadas pela visão biológica e medicinal da alimentação e da nutrição (GAGLIANONE et al., 2006; NASCIMENTO et al., 2011).

Em geral, as pesquisas relatam a visão de profissionais que estão fora do espaço escolar, sobretudo, porque ainda são poucos os trabalhos realizados nas escolas por educadores e professores.

Nota-se ainda que há falta de preparo dos professores e das escolas para tratar a temática (FERNANDES et al., 2005; PRECIOSO, 2009; ZANCUL e COSTA, 2012) e que os livros didáticos também apresentam predominantemente um enfoque biológico da alimentação (DINIZ et al., 2010; FIORE et al, 2012; GREENWOOD e FONSECA, 2016).

Considerando o panorama exposto, esse trabalho tem o objetivo de questionar e propor uma reflexão a respeito da maneira como a Educação Alimentar escolar vem sendo tratada, ressaltando a importância de atuações e práticas que extrapolem a abordagem exclusivamente biológica.

\section{A educação alimentar na escola}

A Educação Alimentar é definida de diferentes formas por distintos pesquisadores, de acordo com Gobbi (2005), é uma parte importante da educação em saúde e é fundamental para a formação de conceitos e atitudes relacionadas à boa saúde a longo prazo. Já Pipitone (1994), considera a educação alimentar como uma prática tradicionalmente desenvolvida com base na integração entre educação e saúde. Rodrigo e colaboradores (2013) definem a Educação Alimentar como um conjunto de métodos e estratégias que ajudam indivíduos e populações a terem uma alimentação saudável.

No que diz respeito à Educação Alimentar na escola as atuais políticas públicas de alimentação e nutrição no Brasil, de acordo com Amparo-Santos (2013), reconhecem a importância da Educação Alimentar como estratégia de promoção da alimentação saudável principalmente dentro do contexto da realização do direito humano a alimentação adequada e da garantia da segurança alimentar. E essas políticas vão além 
do oferecimento de alimentos na merenda escolar, que no Brasil, ocorre na Rede Pública de Ensino em todo o país desde 1955 (BRASIL, 2009).

Existem também documentos oficiais que têm debatido a temática, como por exemplo, a publicação lançada em parceria com o Ministério da Saúde, da Portaria Interministerial $n^{\circ} 1.010$, de 8 de maio de 2006, que institui as diretrizes para a alimentação saudável nas escolas, além da instituição do Programa de Saúde na Escola (PSE) pelo Decreto $n^{\circ}$ 6.286/2007, que visa ações de prevenção e promoção à saúde também incluindo alimentação saudável (SANTOS, 2012)

Em novembro de 2012, foi publicado o Marco de Referência de Educação Alimentar e Nutricional para Políticas Públicas (BRASIL, 2012), este documento orienta conceitos e diretrizes que visam contribuir para a construção de práticas a serem traduzidas em eixos, ações e programas mais qualificados de Educação Alimentar (AMPARO-SANTOS, 2013). O documento indiscutivelmente é relevante, no entanto, é necessário ampliar a discussão sobre as suas possibilidades, seus limites e o modo como são realizados os programas (RAMOS et al, 2013).

Apesar dos avanços recentes na área, o campo da Educação Alimentar no Brasil ainda não é bem definido, especialmente no contexto escolar, existem poucas referências teóricas e metodológicas para subsidiar as práticas (PACHECO, 2008).

Em relação a ações, intervenções e pesquisas desenvolvidas a respeito de alimentação escolar no Brasil, Silva e Fonseca (2009), analisaram artigos científicos publicados entre 2000 e 2008 e constataram uma baixa produção científica sobre a temática e identificaram que nas ações educativas houve predomínio de abordagens teórico-metodológicas tradicionais, em geral, de caráter informativo, e baseadas na transmissão de conhecimento.

Seguindo essa mesma linha, em trabalho que descreve e analisa o panorama de publicação científica sobre estudos de intervenção no campo da Educação Alimentar em escolares no Brasil, Ramos et al., (2013) apontam nos resultados que há poucas publicações na área, que os estudos são, em sua maioria, superficiais, e recomendam a necessidade de metodologias alternativas e inovadoras para abordar a temática.

Analisando os artigos relacionados à Educação Alimentar publicados nas Atas dos I ao VI Encontro Nacional de Pesquisa em Educação em Ciências (ENPEC), Oliveira e Augusto (2009), encontraram seis trabalhos, indicando que esta temática no ensino de Ciências é recente e pouco abordada. Além disso, os artigos analisados sugerem a necessidade das escolas tratarem de forma mais sistemática a educação 
alimentar e de cursos de formação de professores que possibilitem um entendimento mais complexo e interdisciplinar das noções implicadas nos conceitos de alimentação.

De acordo com Motta e Teixeira (2012), a abordagem das questões alimentares no cotidiano escolar considera somente os aspectos biológicos e o valor nutritivo dos alimentos, restringindo a compreensão da alimentação apenas como necessária para a manutenção da saúde. No entanto é fundamental que haja uma compreensão de que, segundo Loureiro (2004), qualquer intervenção num campo específico, como a educação alimentar, não pode restringir-se à comida e muito menos a nutrientes.

O comportamento alimentar é demasiadamente complexo e pode ser justamente por isso que a Educação Alimentar na escola seja reduzida, na maioria das vezes, apenas ao ponto de vista da nutrição, e isso é ruim, já que a nutrição é só um dos diversos condicionantes do comportamento alimentar (ZAFRA, 2011).

A situação é inquietante, se a Educação Alimentar é um tema complexo e amplo, por que aparece na escola de maneira tão reduzida? A Educação Alimentar que vem sendo realizada nas escolas, em geral, não dialoga com as necessidades, anseios e dúvidas dos estudantes nem com a maneira pela qual a sociedade atualmente tem se relacionado com os alimentos.

Sobretudo é imperativo, na qualidade de educadores, refletirmos a respeito das possibilidades existentes para extrapolar as abordagens biológicas da questão, principalmente no ambiente escolar, considerando os diversos aspectos tão fundamentais, como questões sociais, emocionais, econômicas, culturais entre outras, que se associam ao ato alimentar.

\section{Conceito e prática para além da abordagem biológica}

Um fato que chama a atenção é que nas escolas e nos documentos governamentais, a Educação Alimentar é frequentemente apresentada como uma ferramenta para "combater" o aumento da Obesidade e Doenças Crônicas associadas, e embora essa seja uma consideração importante, não pode ser a única que justifique uma alimentação saudável. Em seu provocativo livro "Em defesa da comida: um manifesto", Pollan (2008), afirma que é difícil concluir que a alimentação científica tenha nos deixado mais saudáveis, já que a campanha em favor da pouca gordura coincidiu com 
um drástico aumento da obesidade e diabetes nos EUA. O que significa enfoque meramente biológico pode, de fato, nos afastar de uma alimentação mais saudável.

Nesse sentido é preciso que os professores assumam uma postura critica a respeito do que vem sendo apresentado e da maneira como a Educação Alimentar tem sido tratada nas escolas.

A análise do comportamento alimentar apenas sob a ótica da saúde e doença desconsidera a complexa natureza da alimentação e da cultura. $\mathrm{O}$ ato alimentar não é só biológico como propõe a visão mecanicista do corpo humano. A saúde é apenas um aspecto de motivação do comportamento alimentar que é condicionado por uma série de outras variáveis revestidas de distintos significados (GARCIA-ARNAIZ, 2007).

A pesquisadora Sophie Deram faz uma analise interessante dos significados do ato alimentar e da relação que a sociedade tem estabelecido com os alimentos em seu livro "O peso das dietas" (2014). Ela também ressalta que a escola tem um papel importante na Educação Alimentar e que uma alimentação saudável deve ser variada, equilibrada e consumida com prazer e com atitudes adequadas, por exemplo, comer sem culpa, o que significa que o comportamento é tão importante quanto o nutriente.

Essas são demonstrações de que há pesquisadores pensando na alimentação para além da biologia. As ações de Educação Alimentar na escola podem extrapolar a abordagem biológica do ato alimentar, sugerindo reflexão e debate para uma nova maneira da sociedade se relacionar com os alimentos e se questionar.

Por exemplo, quando trabalhamos com Educação Alimentar na escola é essencial considerar o relevante papel da mídia e problematizar o discurso midiático com os estudantes. Para Teo (2010), a mídia difunde padrões estéticos corporais e alimentares, interferindo nas escolhas feitas e contribuindo para um saber comum sobre práticas alimentares que é frágil e não habilita as pessoas a escolhas saudáveis.

Uma proposição que vem sendo defendida por pesquisadores espanhóis, entre os quais Aranceta Bartrina (2008), é tratar da alimentação dentro da escola para se trabalhar educação alimentar. Segundo o autor, a merenda escolar e a cantina desempenham funções nutricionais que satisfazem não só as necessidades orgânicas, mas também funções educativas, contribuindo para a criação de hábitos alimentares que favoreçam o desenvolvimento e a promoção da saúde. Desse modo, a escola deveria utilizar tanto a merenda como a cantina escolar para ampliar o restrito repertório dos alimentos consumidos pelos jovens. Além desses aspectos, Zafra (2011) aponta que a 
cantina pode ser aproveitada com cunho pedagógico para serem trabalhados aspectos sociais e culturais da alimentação.

Para Loureiro (2004), a escola deve ser um local que ofereça opções de alimentos saudáveis e saborosos, e que as mensagens aprendidas em aulas estejam em consonância com os produtos alimentares disponíveis.

A intenção deve ser de uma Educação Alimentar que ensine a comer no sentido amplo do seu significado, que vai além da transmissão de conhecimentos nutricionais, considerando também questões emocionais, sociais, culturais. A educação alimentar baseada em novas ideias educativas e adaptada à realidade das pessoas com quem pretende atuar e sendo assim, uma Educação Alimentar que considere a diversidade dos contextos alimentares existentes (ZAFRA, 2012).

Podemos concluir que a escola é um local privilegiado para se educar em saúde, e isso se faz de modo mais efetivo por meio da prática.

\section{Considerações finais}

Vivemos um momento marcado por excessos e privações alimentares, em que a mídia dita comportamentos e impõem padrões estéticos. Se quisermos, na qualidade de educadores, que crianças e jovens possam desenvolver uma relação mais feliz e saudável com a alimentação precisamos repensar a forma de abordagem das questões alimentares nas escolas.

É primordial o investimento na formação docente e modificações nos materiais escolares no sentido de aprofundar a discussão e a compreensão de que a Educação Alimentar extrapola questões biológicas e se relaciona profundamente a aspectos emocionais, sociais, econômicos, culturais e comportamentais como, por exemplo, a afetividade e outras questões emocionais que nos levam a comer além da fome e além do ato de nutrir o corpo.

AGRADECIMENTOS: Este artigo foi parcialmente apoiado por uma Bolsa de Estágio Pós-Doutoral da Coordenação de Aperfeiçoamento Pessoal de Nível Superior-CAPES. 


\section{REFERÊNCIAS}

AMPARO-SANTOS, L. Avanços e desdobramentos do marco de referência da educação alimentar e nutricional para políticas públicas no âmbito da universidade e para aspectos culturais da alimentação. Revista de Nutrição, Campinas, v.26, n.5, p.595-600, 2013.

ARANCETA BARTRINA, J. Realidad actual de los comedores escolares en España. In: MARTÍNEZ ALVAREZ, J. R.; POLANCO ALLUÉ, I. (coord.). El libro blanco de la alimentación escolar. Madrid: McGraw-Hill, 2008.

BRASIL. Ministério da Educação e Cultura. Secretaria de Educação Fundamental. Parâmetros curriculares nacionais: terceiro e quarto ciclos: apresentação dos temas transversais. Brasília, DF, 1998.

BRASIL. Alimentação escolar. MEC/FNDE. 2009.

BRASIL. Marco de referência de educação alimentar e nutricional para as políticas públicas. Brasília, DF: MDS; Secretaria Nacional de Segurança Alimentar e Nutricional, 2012.

CARNEIRO, H. Comida e sociedade: uma história da alimentação. Rio de Janeiro: Campus, 2003.

CASTELL, G. S. Larousse da dieta e da nutrição. São Paulo: Larousse do Brasil, 2004.

COELHO, R. et al. Excesso de peso e obesidade prevenção na escola. Acta Med Port, v.21, p. 341-344, 2008.

DERAN, S. O peso das dietas: emagreça de forma sustentável dizendo não às dietas! São Paulo: Sensus, 2014.

DINIZ, M. C. P.; OLIVEIRA, T. C.; SCHALL, V. T. Saúde como compreensão de vida: avaliação para inovação na educação em saúde para o ensino fundamental. Revista Ensaio, v.12, n.01, p.119-144, 2010.

FERNANDES, M. H.; ROCHA, V. M.; SOUZA, D. B. A concepção sobre saúde do escolar entre professores do ensino fundamental $\left(1^{\mathrm{a}}\right.$ a $4^{\mathrm{a}}$ séries $)$. História, Ciências, Saúde - Manguinhos, v. 12, n. 2, p. 283-291, 2005.

FIORE E. G.; JOBSTRAIBIZER, G. A.; SILVA, C. S.; CERVATO-MANCUSO, A. M. Abordagem dos Temas Alimentação e Nutrição no Material Didático do Ensino Fundamental: interface com segurança alimentar e nutricional e parâmetros curriculares nacionais. Saúde e Sociedade, São Paulo, v.21, n.4, p.1063-1074, 2012.

GAGLIANONE C. P. et al. Nutrition education in public elementary schools of São Paulo, Brazil: the Reducing Risks of Illness and Death in Adulthood project. Revista de Nutrição, Campinas, v.19, n.3, p.309-320, 2006. 
GARCIA-ARNAIZ, M. Comer bien, comer mal: la medicalización del comportamiento alimentario. Salud Pública de México, v. 49, n. 3, p. 236-242, 2007.

GOBBI, L.S. A educação nutricional para a prevenção da obesidade infantil em uma instituição particular de ensino do município de Bauru - SP. 2005. Dissertação (Mestrado) Faculdade de Ciências Farmacêuticas. - UNESP, Araraquara, 2005.

GREENWOOD, S. A.; FONSECA, A. B. Espaços e caminhos da educação alimentar e nutricional no livro didático. Ciências. Educação, Bauru, v. 22, n. 1, p. 201-218, 2016.

LYNG, N. et al. I. Reporting accuracy of packed lunch consumption among Danish 11year-olds differ by gender. Food \& Nutrition Research, v.57: 19621, 2013.

LOUREIRO, I. A importância da educação alimentar: o papel das escolas promotoras de saúde. Revista Portuguesa de Saúde Pública, v.22, n.2, p.43-55, 2004.

MOTTA, M. B.; TEIXEIRA, F. M. Educação Alimentar na escola por uma abordagem integradora nas aulas de Ciências. Inter- Ação, v. 37, n.2, p.359-379, 2012.

NASCIMENTO, A. M.; MAGALHÃES, M. C.; PAES, M. S. Enfermeiro e escola: uma parceria na prevenção da obesidade infantil. Revista Enfermagem Integrada, Ipatinga, v.4, n.1, p.742-754, 2011.

OLIVEIRA, G. M. S. M.; AUGUSTO, T. G. S. Análise dos artigos sobre Educação Alimentar publicados nas Atas do ENPEC. In: Anais do VII Encontro Nacional de Pesquisa e Ensino de Ciências. Florianópolis: ABRAPEC, 2009.

PACHECO, S. S. M. O hábito alimentar enquanto um comportamento culturalmente produzido. In: FREITAS, M. C. S.; FONTES, G. A.V.; OLIVEIRA, N. (org.). Escritas e narrativas sobre alimentação e cultura, Salvador, EDUFBA, 2008.

PIPITONE, M. A. P. A relação saúde educação na Escola de 1o grau. Rev. Alim. Nutr., v. 65, p. 48-52, 1994.

POLLAN, M. Em defesa da comida: um manifesto. Rio de Janeiro: Intrínseca, 2008.

PRECIOSO, J. As Escolas Promotoras de Saúde: uma via para promover a saúde e a educação para a saúde da comunidade escolar. Educação, Porto Alegre, v.32, n.1, p.8491, 2009.

RAMOS, F. P.; SANTOS, L. A. S.; REIS, A. B. C. Educação alimentar e nutricional em escolares: uma revisão de literatura. Cad. Saúde Públ., v.29, n. 11, p. 2147-2161, 2013.

RODRIGO, M. V.; EJEDA, J. M. M.; ARMENTA, M. C. Una década enseñando e investigando en Educación Alimentaria para Maestros. Revista complutense de educación, v. 24, n. 2, p. 243-265, 2013.

SANTOS, L. A. S. O fazer educação alimentar e nutricional: algumas contribuições para reflexão. Ciência \& Saúde Coletiva, v.17, n. p.453-462, 2012. 
SILVA, E. C. R.; FONSECA, A. B. Abordagens pedagógicas em Educação Alimentar e Nutricional em escolas do Brasil. In: Anais do VII Encontro Nacional de Pesquisa e Ensino de Ciências. Florianópolis: ABRAPEC, 2009.

TEO, C. R. P. A. Discursos e a construção do senso comum sobre alimentação o a partir de uma revista feminina. Saúde e Sociedade, São Paulo, v.19, n.2, p.333-346, 2010.

ZAFRA, E. La transversalidade de los processos de socialización en los comportamentos alimentarios de los niños y niñas. Nuevas propuestas para la educación alimentaria. Zainak, Cuadernos de Antropología-Etnografía, v. 34, p.33-60, 2011.

ZAFRA, E. Experiencias de aprendizaje alimentario desde lo corporal. Nuevas propuestas para la educación alimentaria. In: GARCIA- ARNAIZ, M. Alimentación, salud y cultura: encuentros interdisciplinares. Tarragona: URV, 2012. p. 41-60.

ZANCUL, M. S.; COSTA, S. S. Concepções de professores de ciências e de biologia a respeito da temática educação em saúde na escola. Experiências em Ensino de Ciências, v.7, n.2, p.67-75, 2012.

\section{Como referenciar este artigo}

ZANCUL, Mariana de Senzi. Educação alimentar na escola: para além da abordagem biológica. Temas em Educ. e Saúde, Araraquara, v.13, n.1, p. 14-23, jan./jun. 2017. DOI <https://doi.org/10.26673/rtes.v13.n1.jan-jun2017.2.9490>. ISSN: 1517-7947.

Submetido em: 02/03/2017

Aprovado em: 20/08/2017 